\title{
Schnarchprothesen verbessern die kognitive Funktion
}

\author{
In bestimmten Fällen können sog. \\ Schnarchprothesen bei Schlafapnoe \\ das bessere Therapieverfahren im \\ Vergleich zur CPAP-Therapie darstel- \\ len.
}

— Die obstruktive Schlafapnoe (OSA) führt durch eine Störung des Schlafes zu vermehrter Tagesmüdigkeit und Einschränkung der geistigen Leistungsfähigkeit. Der "Goldstandard" der OSABehandlung, die CPAP (continuous positive airway pressure)-Therapie, beseitigt diese Symptomatik sicher. Sind aber auch alternative Therapieverfahren wie die sog. Schnarchprothesen in der Lage, die Tagessymptomatik der OSA-Patienten zu beeinflussen?

Die Verbesserung der mit neurophysio- und psychologischen Methoden messbaren kognitiven Funktionen von OSA-Patienten durch die CPAP-Therapie ist in der Literatur gut belegt. Bei leicht bis mittelgradig ausgeprägter OSA werden sog. Schnarchprothesen bzw. Schnarchschienen (Unterkieferprotrusions-Schienen = UKPS) als therapeutische Alternative zur CPAP-Therapie

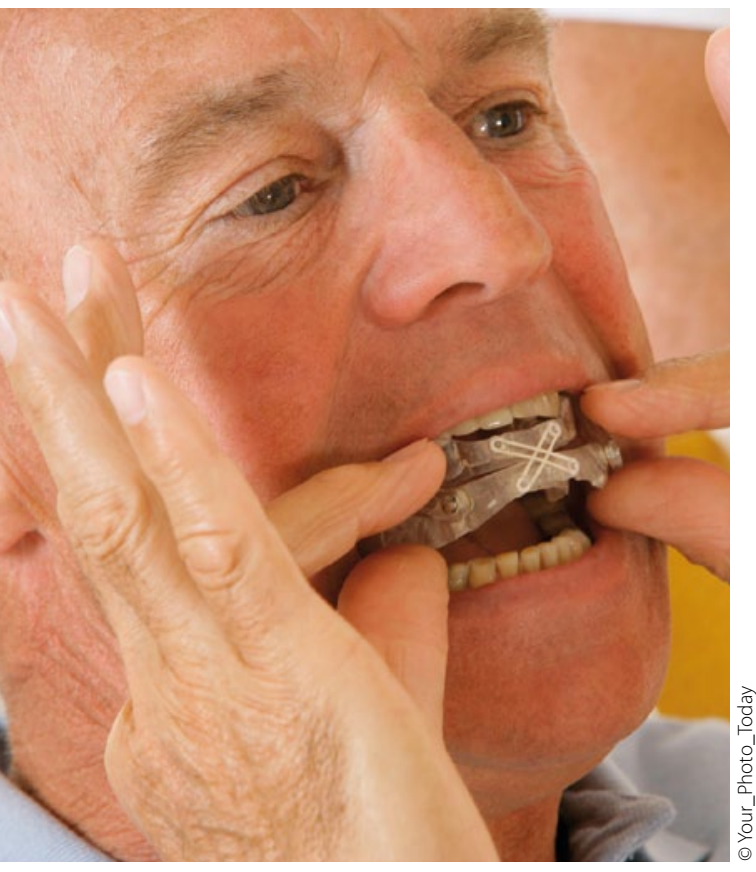

Schnarchprothesen können eine Alternative zur CPAP darstellen. angewandt. Es gibt aber bisher nur eine kontrollierte randomisierte Studie mit einem positiven Effekt der Schnarchprothesen auf Vigilanz und psychomotorische Funktionen der behandelten $\mathrm{Pa}$ tienten Die referierte Arbeit untersuchte daher den Effekt der Behandlung von OSA-Patienten mit einer Schnarchprothese auf verschiedene kognitive Funktionen.

In die prospektive Studie wurden 50 männliche OSA-Patienten einer HNOKlinik in Uppsala/Schweden eingeschlossen. Das Alter der Patienten lag zwischen 20 und 65 Jahren. Sie wiesen einen polysomnografisch bestimmten Apnoe-Hypopnoe-Index (AHI) von mindestens 10 auf (leichte OSA 10-20, mäßiggradige OSA 20-30, schwere OSA $>30$ ).

Die Patienten wurden zu Beginn der Studie und nach sechs Monaten Therapie polysomnografiert. Zeitgleich erfolgte jeweils eine ausführliche neuropsychologische und neurophysiologische Testung (u.a. Working Memory Test, Continuous Performance Test, Trail-Making Test).

Der AHI sank unter Therapie von $40.9 \pm 16.0$ auf $18.0 \pm 17.3(\mathrm{p}<0.001)$. Auch bei schwerer Schlafapnoe (AHI > 30) wurde der AHI im Mittel um 50\% gesenkt ( $\mathrm{p}<0,001) .67 \%$ der Patienten mit mittelgradig ausgeprägter und $57 \%$ mit schwergradig ausgeprägter OSA sprachen auf die Therapie an. Der Epworth-Sleepiness-Scale-(ESS)-Score betrug vor Therapie 11,2 $\pm 4,1$ und war somit leichtgradig pathologisch erhöht. Nach sechsmonatiger Therapie konnte eine Reduktion in den Normbereich auf $6,8 \pm 4,5$ erzielt werden. Die Reduktion des ESS-Scores war signifikant $(\mathrm{p}<$ 0,001 für Patienten mit normalisierter Atmung im Schlaf, $\mathrm{p}<0,02$ für Patienten mit noch teilweise vorhandener pathologischer Atmung im Schlaf).

Alle Bereiche der getesteten kognitiven Funktionen verbesserten sich nach sechsmonatiger UKPS-Therapie ( $\mathrm{p}<$ 0,001). Dieser Effekt war auch bei schwergradiger OSA abgeschwächt nachweisbar. Insbesondere kam es zu einer Verbesserung der Vigilanz bzw. Daueraufmerksamkeit sowie der motorischen und mentalen Schnelligkeit. Es bestand keine bzw. keine enge Korrelation zu den Veränderungen der Atmungsparameter im Schlaf.

\section{- A. Tegelberg et al. \\ Improved cognitive functions after treatment with oral appliance in obstructive sleep apnea. Nature and Science of Sleep 2012; 4: 89-96}

\section{Kommentar}

Die referierte Studie befasst sich mit einem alten, aber immer wieder neuen Thema der Schlafmedizin, nämlich dem Nutzen von Schnarchprothesen bei Schlafapnoe. Schnarchprothesen zur Behandlung der Schlafapnoe gibt es schon länger als die CPAP-Therapie. Der anfänglich unkontrollierte und mit fehlendem schlafmedizinischem Hintergrund erfolgte Einsatz dieses Therapieverfahrens führte über viele Jahre zu einer breiten Negativeinschätzung durch die Schlafmediziner. Diese Entwicklung gipfelte in einer Streichung der Schnarchprothesen aus dem Heil-und Hilfsmittelkatalog, sodass derzeitig nur Einzelfallentscheidungen der jeweiligen Krankenversicherung im Hinblick auf eine Therapiekostenübernahme möglich sind.

Die zahnmedizinische Schlafmedizin hat aber mittlerweile ihre "Hausaufgaben" ausgezeichnet erledigt. Technisch ausgefeilte Unterkieferprotrusionsschienen, das Bekenntnis der Zahnmediziner zur prätherapeutischen Anwendung der Prinzipien der schlafmedizinischen Diagnostik und mittlerweile weitreichende Erfahrung in der richtigen Indikationsstellung sowie die enge Vernetzung mit klinischer und wissenschaftlicher Schlafmedizin haben dazu geführt, dass in bestimmten Fällen die Schnarchprothese sogar das bessere Therapieverfahren im Vergleich zur CPAP-Therapie darstellen kann. Schnarchprothesen müssen daher zukünftig vielmehr als bisher geschehen mit in das therapeutische Management der OSA einbezogen werden. Hierzu müssen Schlafmediziner und Zahnmediziner noch enger zusammenrücken.

K. RASCHE " 